Document downloaded from:

http://hdl.handle.net/10251/104546

This paper must be cited as:

Molina Mateo, J.; Salmerón Sánchez, M.; Monleón Pradas, M.; Torregrosa Cabanilles, C. (2012). Multilayer adsorption by Monte Carlo simulation. Physica A Statistical Mechanics and its Applications. 391(20):4474-4782. doi:10.1016/j.physa.2012.05.016

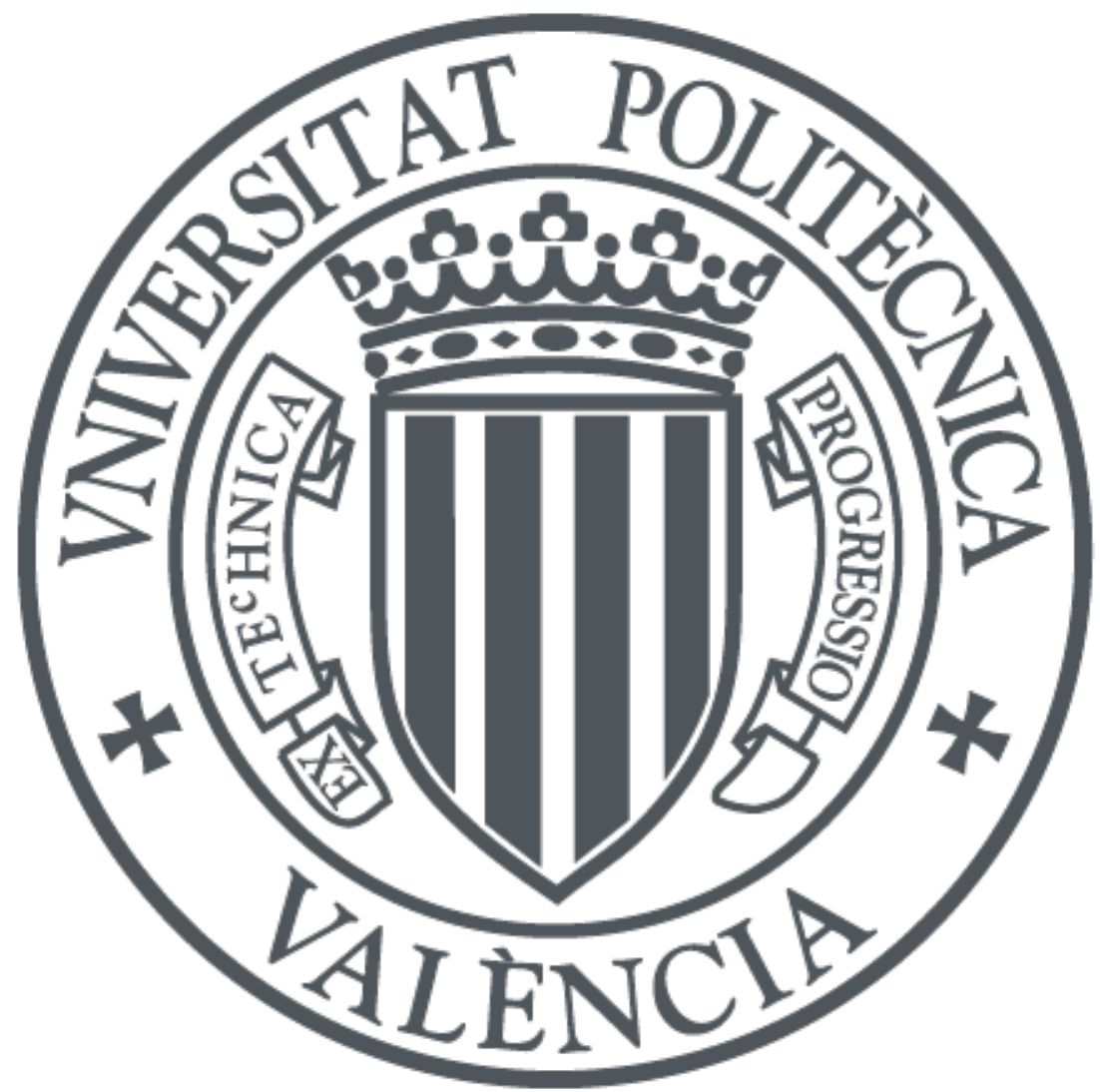

The final publication is available at

https://doi.org/10.1016/j.physa.2012.05.016

Copyright Elsevier

Additional Information 


\title{
Multilayer Adsorption by Monte Carlo Simulation
}

\author{
J. Molina-Mateo ${ }^{\mathrm{a}}$, M. Salmerón Sánchez ${ }^{\mathrm{a}, \mathrm{b}}$, M. Monleón Pradas ${ }^{\mathrm{a}, \mathrm{b}}$, \\ C. Torregrosa Cabanilles ${ }^{\text {a,* }}$ \\ ${ }^{a}$ Centre de Biomaterials i Enginyeria Tissular, Universitat Politècnica de València, \\ 46022, Cami de Vera 14, València, Spain \\ ${ }^{b}$ Networking Research Center on Bioengineering, Biomaterials and Nanomedicine
}

(CIBER-BBN), València, Spain

\begin{abstract}
Adsorption phenomena are characterized by models that include free parameters trying to reproduce experimental results. In order to understand the relationship between the model parameters and the material properties, the adsorption of small molecules on a crystalline plane surface has been simulated using the Bond Fluctuation Model. A direct comparison between the Guggenheim-Anderson-de Boer (GAB) model for multilayer adsorption and computer simulations allowed us to establish correlations between the adsorption model parameters and the simulated interaction potentials.

Keywords: Bond Fluctuation Model, multilayer adsorption

PACS: 68.08.De, 05.10.Ln, 05.70.Np
\end{abstract}

\section{Introduction}

The best known adsorption equations, i.e. those of Langmuir, BrunauerEmmett-Teller (BET), Guggenheim-Anderson-de Boer (GAB) have been used

\footnotetext{
${ }^{*}$ Corresponding author.

Email address: ctorregr@fis.upv.es (C. Torregrosa Cabanilles)
} 
to characterize adsorption phenomena in a broad range of fields which include polymer hydrogels and food engineering $[1,2,3,4,5]$. These equations actually characterize equilibrium states, and relate some measure for the composition of the system with the activity or fugacity of the adsorbed species. In fact, all these equations have been rederived mechanostatistically from equilibrium considerations. Moreover, these equations treat the adsorption phenomenon as a problem of 'localizing' the adsorbate molecules onto an adsorbent lattice. This feature delivers a first parameter of physical relevance for these models, the monolayer capacity $w_{m}$, that is a measure (mol number, mass fraction, volume or normalized volume, concentration) of the amount of adsorbate that saturates the available sorption sites, one adsorbed molecule per each sorption site. The total amount of adsorbed molecules can be referred to $w$ through

$$
\theta=\frac{w}{w_{m}}
$$

where $w$ is a measure of adsorbed molecules and $\theta$ is the adsorbed fraction.

However, equations which account only for this feature (e.g. Langmuir's) cannot describe all types of adsorption curves found experimentally. The BET equation introduces a second fundamental notion in the theories of adsorption: the concept of multilayer adsorption, i.e., the idea that the molecules can be adsorbed as piles of more than one unity at each sorption site. Correspondingly, the BET equation contains a second parameter $c$ that accounts for the difference in the energy of the molecules sorbed between the first and other layers [6],

$$
\theta=\frac{c a}{(1-a)[1+(c-1) a]}
$$


The BET equation has nonetheless a number of shortcomings, e.g. it predicts an infinite sorption at activity one, which in due time prompted proposals of better equations. One of them was the GAB equation $[7,8]$,

$$
\theta=\frac{c f a}{(1-f a)[1+(c-1) f a]}
$$

which replaces the activity $a$ in BET by $f a$ and thus has the BET equation as a particular case when $f=1$. This equation has a number of theoretical advantages over the BET equation and also describes more adequately experimental data. Both the BET and the GAB equations have now classical kinetic $[6,7,9]$ and statisticomechanical derivations [10] which link the fitting parameters $c$ and $f$ with well identified physical concepts related to the interactions of sorbate and substrate. Traditionally, the parameter $c$ is related to the energy of the adsorbate in the first adsorption layer, $e_{1}$, and in the second and subsequent layers, $e_{2}$, as

$$
c=c_{0} \exp \frac{e_{2}-e_{1}}{k T}
$$

While the BET equation assumes that $e_{2}$ equals the energy of the pure sorbate in the saturated state, the GAB equation provides the parameter $f$ to set its value

$$
f=f_{0} \exp \frac{e^{o}-e_{2}}{k T}
$$

where $e^{o}$ is the energy in some chosen reference state, e.g. the liquid state.

As soon as the GAB equation is used to extract some information on the nature of the energetic interactions involved in the adsorption process, the preexponential coefficients $c_{0}$ and $f_{0}$ come into play and it is mandatory to calculate their values to get precise information on the energy of 
the adsorption process. Nevertheless, $c_{0}$ and $f_{0}$ are usually assumed to be close to unity without further justification, which is by other means also a controversial issue $[11,12,13,14]$.

In [15] a thermodynamic derivation of the GAB equation was proposed, from which the meaning of $c$ and $f$, including the preexponential factors, is unambiguously obtained, and related to the entropy parameters of the sorbate. Furthermore, during the derivation process were obtained equations giving the number of molecules adsorbed in the first and subsequent layers. That is to say, the fraction of empty sites results to be

$$
\theta_{0}=\frac{1-q}{1-q+c q}
$$

with $q=f a$. While the fraction number of sorption sites occupied by imolecules is sequentially obtained from

$$
\theta_{i}=c \theta_{0} q^{i}, \quad i=1,2,3 \ldots
$$

And also the fraction of molecules directly in contact with the substrate,

$$
\xi(1)=1-\theta_{0}=\frac{c f a}{1+(c-1) f a} .
$$

The thermodynamical derivation of the GAB equation allows one to get a molecular picture of the equilibrium adsorption process at different activities and its energetic and entropic parameters, rather than staying at the mere phenomenological parameters $c$ and $f$. As a consequence, the comparison with computational simulations of the adsorption process can be done at the molecular level. 


\section{Model Hypothesis and Simulations}

The Bond Fluctuation Model $[16,17]$ is one of the most commonly used lattice models employed in order to simulate polymeric materials as well as many physical phenomena such as adsorption $[18,19,20]$. Although it was originally designed to simulate the behavior of high molecular weight systems, this model can simulate any type of substance, including low molecular weight ones. This is why it was chosen to carry out simulations: initially with a simple simulated sorbate for direct comparison with the GAB model, but with the immediate option to increase the molecular weight of the sorbate to investigate adsorption of macromolecules in future works.

The Bond Fluctuation Model (BFM) consists of molecular groups linked by bonds whose length can vary between 3 and $\sqrt{10}$ times the unit cell edge length. Each molecular group is composed of a cube that occupies eight vertexes of the lattice simulation. In this paper we will use only simple molecular groups without any link between them to simulate a low molecular weight substance. The adsorption surface is composed by strongly bonded molecular groups (forming a crystal), packed at the highest density in a cubic lattice.

One step of the Monte Carlo simulation consists of choosing randomly both a molecular group and one of its six possible movements. The movement is carried out as long as it respects the rules of the model, considering the volume exclusion. In order to take into account the energy and the temperature of the system, the movement is finally performed with a probability 
that is given by the Metropolis criterion [21]:

$$
P=\min \left\{1, \exp \left(\frac{-\Delta E}{k T}\right)\right\},
$$

where $\Delta E$ is the increase in energy due to the movement and $T$ is the temperature of the system. The interactions between molecular groups of the system are governed by Lennard-Jones potentials, for both sorbate-sorbate interaction and sorbate-surface interactions [17]

$$
U_{L J}=4 \varepsilon\left\{\left(\frac{\sigma}{r}\right)^{12}-\left(\frac{\sigma}{r}\right)^{6}\right\}
$$

where $\sigma=1.8$. The potential was calculated up to a distance $r_{c}=4$ lattice units. The sorbate-sorbate parameter $\varepsilon$ was fixed to 1 for all simulations, while the $\varepsilon$ parameter for the sorbate-surface interaction took values $\varepsilon=$ $0.5,1,3$ and 5 .

The dimensions of the adsorption surface were $40 \times 40$ lattice units with periodic boundary, which allowed a total of 400 molecular groups in the first adsorbed layer. Three different concentrations of the sorbate where simulated, $\Phi=0.5, \Phi=0.3$ and $\Phi=0.1$. All simulations were conducted with 25200 molecular groups, rescaling the $\mathrm{Z}$ dimension to achieve the desired concentration. For $\Phi=0.5, \mathrm{Z}$ took a value of 240 , for $\Phi=0.3, \mathrm{Z}$ was 400 and for $\Phi=0.1, \mathrm{Z}$ had a value of 1200 units of the lattice. Thus, keeping constant the number of molecular groups at a high value, each completely adsorbed layer means $1.59 \%$ of the total molecular groups, so the concentration of the sorbate could be considered constant in every simulation.

Simulated temperatures were $k T=4,3,2,1.7,1.5$ and 1.3. All these temperatures kept the system in thermodynamic equilibrium, making the 
simulations comparable with GAB model. No lower temperatures were chosen because they were out of equilibrium for our simulation time.

All simulations consisted of an initial equilibration period of $10^{6}$ Monte Carlo Steps (MCS), followed by a period of $10^{5}$ MCS where average values of the occupation of the layers were calculated. A long equilibration period was chosen because the initial state was taken very far from equilibrium. In fact, for practical reasons we started the simulation with the adsorbate configured in the simulation box as a crystall at the opposite side of the adsorption surface. The estimated autocorrelation of the system energy in equilibrium was around $300 \mathrm{MCS}$, increasing to around $10^{3} \mathrm{MCS}$ for the occupied fraction of the higher adsorbed layers. Due to the fact that each molecular group occupied 8 cells of the lattice forming a cube, it was considered that each adsorbed layer had a size of two units of the lattice, as shown in Fig. 1.

In order to compare simulations with different concentrations, the adsorbed fraction on each layer (up to the 10th layer) was normalized according to

$$
\xi=\frac{d-\Phi}{1-\Phi}
$$

where $d$ was the occupied fraction of the layer and $\Phi$ was the average concentration of the simulation, i.e. the expected equilibrium occupation that should be expected in absence of any absorptive surface, that was taken as a reference.

From the adsorbed fraction $\xi(i)$ for each layer $i$, the fraction of vacant sorption sites $\theta_{0}$ and the fraction number of sorption sites occupied by a column of $i$ molecules, $\theta_{i}$, were calculated as

$$
\theta_{0}=1-\xi(1)
$$




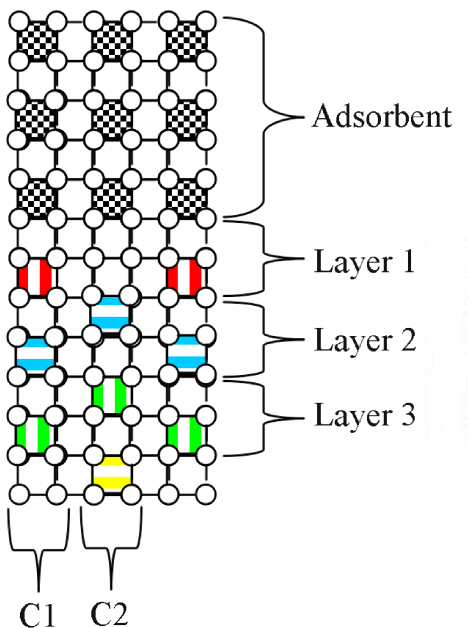

Figure 1: The adsorbent surface was composed of three layers of a Lennard-Jones crystal (black dots). Subsequently, the adsorbed groups were considered belonging to layer 1 (vertical lines, red color), the following layer 2 (horizontal lines, blue color), layer 3 (vertical lines, green color) and so on. Since each molecular group was cubic and occupied 8 vertexes of the lattice, it was considered that each layer should also have the thickness necessary to include a cube as a whole, taking into account the possible offsetting between columns, as in column $1(\mathrm{C} 1)$ and $2(\mathrm{C} 2)$. 
Table 1: Values of the energy parameters calculated from the LJ potentials, and the logarithm of the pre-exponential factor $c_{0}$ obtained from the fitting to simulation results.

\begin{tabular}{ccccc}
\hline$\varepsilon$ & $e^{o}$ & $e_{1}$ & $e_{2}$ & $\ln c_{0}$ \\
\hline 1.0 & -4.9 & -12.1 & -9.2 & -2.1 \\
3.0 & -4.9 & -21.4 & -9.2 & -3.5 \\
5.0 & -4.9 & -30.7 & -9.2 & -5.6 \\
\hline
\end{tabular}

$$
\theta_{i}=\xi(i)-\xi(i+1)
$$

If we consider the system at a maximum concentration $\Phi=0.5$ as a pure liquid, which is the standard density of a liquid in BFM simulations, then the molar fractions $x=0.196,0.594$ and 1 correspond to the selected concentrations $\Phi=0.1,0.3$ and 0.5 respectively.

The comparison between the simulation results and the GAB model requires the calculation of the energy coefficients $e^{o}, e_{1}$ and $e_{2}$ involved in $f$ and $c$ GAB parameters (Eq. 4 and 5). The energy coefficients can be related to the simulation interaction potentials and were physically interpreted in [15] as follows: $e^{o}$ is the energy of a molecular group in conditions chosen as standard, with density 0.5 and activity $a=1$, in the liquid state and was calculated as half the energy of a group into a completely packed region with density $1 ; e_{1}$ is the difference between the energy of a group in the first adsorption layer, when this first layer is complete, and the energy of a vacant, when this first layer is empty; and $e_{2}$ is the averaged energy of a group in the adsorbed layers except the first layer. Depending on the values of the sorbate-surface interaction coefficient $\varepsilon$, the values of those energy coefficients are shown in Table 1. 


\section{Results and Discussion}

\subsection{Simulated Adsorption with Bond Fluctuation Model}

Simulations with different temperatures and concentrations were performed for several fixed values of the $\varepsilon$ parameter of the sorbate-surface interaction. At temperatures $k T<1.3$, the system did not reach the equilibrium state. At $k T=1$, and for either any value of $\varepsilon$ or concentration, a complete multilayer adsorption of 8 or more layers was observed and interpreted as crystallization rather than adsorption. By contrast, no adsorption was observed with $\varepsilon=0.5$, due to the effect of the sorbate-sorbate interaction, that prevailed over the weak attractive interaction potential of the surface. This effect occurred for every equilibrium temperature and concentration.

At equilibrium, when $\varepsilon$ was increased to 1 , the effect of multilayer adsorption was observed (Fig. 2a). As expected, the number of adsorbed layers increased as concentration did. However, even at the highest concentration, the first layer was not completed (jamming). On the other side, the number of adsorbed layers diminished as temperature increased. For temperatures greater than $k T=3$, adsorption was prevented due to the entropic repulsion effect of the wall. It should be noted that, in this case, the sorbate-surface interaction energy was the same as the sorbate-sorbate interaction one. Therefore, the adsorption observed in these simulations was merely a consequence of the steric impact caused by the surface, without any contribution of the interaction energies.

The higher the values of $\varepsilon$, the higher the contribution of energy to adsorption. Compared to the results with lower values of $\varepsilon$, the number of adsorbed layers increased and the first layer was completed (Fig. 2b), which 

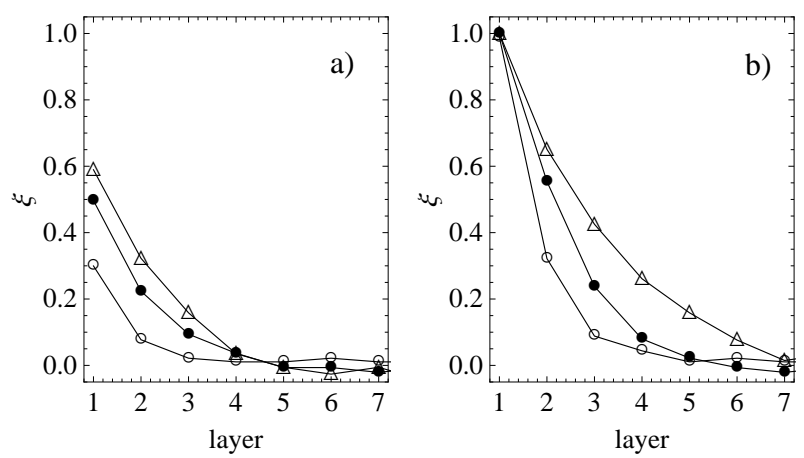

Figure 2: Occupied fraction $\xi$ of each layer obtained from simulations with interaction potential parameters $\varepsilon=1$ (a) and $\varepsilon=3$ (b), with different concentrations $\Phi=0.1$ (o), $0.3(\bullet)$ and $0.5(\triangle)$, at the same temperature $k T=1.3$. Lines are only eyes guides.

even reached 6 adsorbed layers for the highest concentration. Likewise, the number of adsorbed layers increased as concentration did. On the other hand, the number of adsorbed layers diminished as temperature increased and, by contrast, at least one adsorbed layer was found even at the highest simulated temperature $(k T=4)$.

\subsection{Interpretation of the simulated adsorption with the GAB model}

The fraction number of both vacant sorption sites $\theta_{0}$ and sorption sites occupied by a column of one element, $\theta_{1}$, were obtained from the simulation results. These values were related to the GAB model parameters $q=f a$ and $c$ through Eq. 6 and 7 as

$$
\begin{aligned}
c & =\frac{1-q-\theta_{0}+\theta_{0} q}{\theta_{0} q} \\
q & =\frac{\theta_{1}+\theta_{0}-1}{\theta_{0}-1} .
\end{aligned}
$$

Simulations results at different temperatures $k T$ and molar fractions $x$ allowed one to obtain $q(x, T)$ y $c(T)$. Additionally, the GAB model parameter 
$f$ (Eq. 3) is related to $q$, the activity $a=\gamma x$ and the molar fraction $x$ as

$$
q=f \gamma x
$$

where $\gamma$ is the activity coefficient. Simulations of pure liquid $(x=1$ and $\gamma=1$ ) allowed one to calculate $f(T)=q(T)$. For solutions $(x<1)$, the activity coefficient $\gamma$ was obtained from Eq. 16, which as expected was found to depend on temperature but not on the sorbate-surface interaction parameter $\varepsilon$. $\gamma$ was approximately obtained to be 2.5 for $x=0.2$ and 1.3 for $x=0.6$ from a lineal fitting in the temperature range between $k T=1.3$ and $k T=2$. At lower temperatures the system is not in equilibrium, while at higher temperatures fluctuations are too high. The calculated activity coefficients correspond to a positive deviation of the Henry's law. The cohesive interaction between sorbate elements is higher than the adhesive interaction between sorbate and solvent, because the solvent is considered as a non-interacting free volume at the simulation.

\subsubsection{Preexponential factors $c_{0}$ and $f_{0}$}

The temperature dependence of the GAB parameters $c$ and $f$ obtained from simulations in different conditions agrees with that stated by Eq. 4 and 5. Moreover, the parameter $f(T)$ evolves independently of the values assumed for the sorbate-surface interaction parameter $\varepsilon$, as expected from its physical meaning (see Fig. 3). Likewise, according to Eq. 4, $c(T)$ depends strongly on the interaction potential between sorbate and surface (see Fig. 4).

From the expressions for $c(T)$ and $f(T)$ (Eq. 4 and 5), and using the fixed values of the energy parameters $e^{o}, e_{1}$ and $e_{2}$ included in Table 1 , we fit the data with only one free parameter, the preexponential $f_{0}$ (Fig. 3) or 


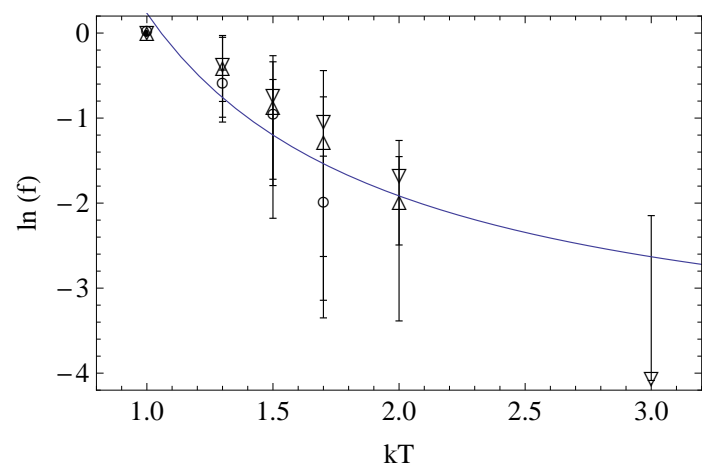

Figure 3: Logarithmic values of $f$ for $\varepsilon=1(\circ), 3(\Delta)$ and $5(\nabla)$, and fitted function (line).

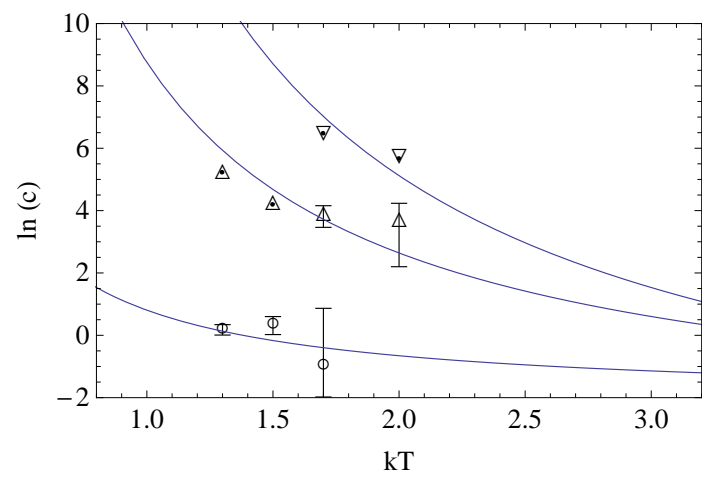

Figure 4: Logarithmic values of $c$ for $\varepsilon=1(\circ), 3(\Delta)$ and $5(\nabla)$, and fitted functions (lines).

$c_{0}$ (Fig. 4). The values of these parameters are found far from being unity, as it is usually assumed in the literature $[4,5,12,13,14]$. That is to say, fittings of $c(T)$ and $f(T)$ to simulation data allows one to obtain $c_{0}$ and $f_{0}$ unambigously. Results show that both $c_{0}$ and $f_{0}<1$, with $\ln f_{0}=-4.1$ and $\ln c_{0}$ shown in Table 1 as a function of the sorbate-surface interaction.

The preexponential parameters $c_{0}$ and $f_{0}$ have been related to the entropy 


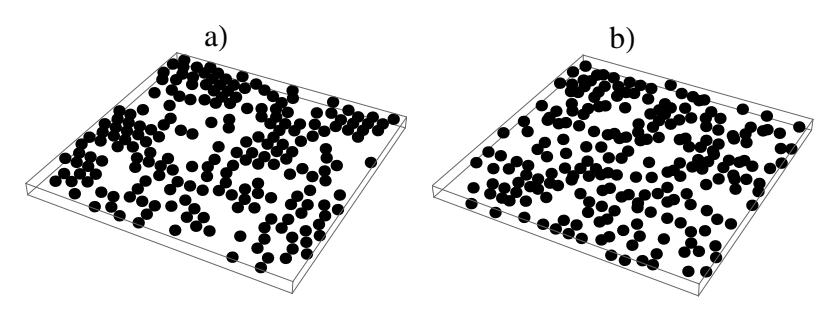

Figure 5: First adsorbed layer with an occupied fracction 0.5 (a) and a typical liquid layer (b), with an equivalent density.

of the sorbate [15],

$$
\begin{aligned}
\ln c_{0} & =\frac{s_{1}-s_{2}}{k} \\
\ln f_{0} & =\frac{s_{2}-s^{o}}{k},
\end{aligned}
$$

where $s^{o}$ is the entropy of a sorbate element in the conditions chosen as standard, with activity $a=1$ (liquid state), $s_{1}$ is the entropy of a molecule in the first adsorption layer when it is completed and $s_{2}$ is the entropy of the sorbed state at intermediate layers.

Since the product $c_{0} f_{0}<1$, the liquid state entropy $s^{o}$ is higher than that of the adsorbed state in the first layer, $s_{1}$, which means that the adsorbed state is more ordered than the liquid one, as shown in Fig. 5.

Since for every calculated preexponetial factor $c_{0}$ holds $c_{0}<1$, the entropy of the molecules directly in contact with the substrate, the first layer, $s_{1}$ is lower than that of the intermediate layers, $s_{2}$. Moreover the $c_{0}$ values shown in Table 1 depend on the sorbate-surface interaction potential $\varepsilon$ through the entropy of the first layer $s_{1}$. As the interaction is stronger, the entropy $s_{1}$ decreases and the molecular order of the first adsorbed layer increases. 


\subsubsection{Comparison between $G A B$ and Monte Carlo simulations}

As a measure of the agreement between simulations and the GAB model, results of the simulated multilayer adsorption and that of the GAB model fitted to simulated adsorption are compared. The total adsorbed fraction $\theta$ (Fig. 6) and the fraction number of sorption sites occupied by a column of $i$ molecules, $\theta_{i}$ (Fig. 7), were obtained from both computer simulations and the GAB model using (i) the energy values related to the LJ potentials (Table 1 ), (ii) the activity coefficients and (iii) the values of $c_{0}$ and $f_{0}$ previously calculated.

The GAB model is able to reproduce the adsorbed fraction calculated after simulations for several sorbate-surface interaction strengths and different concentrations of the sorbate (see Fig. 6) and in a broad temperature interval, between $k T=1.3$ and $k T=2$. By contrast, the extrapolation is not accurate at higher temperatures (when $k T>2$ ), due to the aforementioned difficulties for the estimation of the activity coefficient.

Qualitatively good agreement is also found between predictions of the simulations and the GAB model for the fraction number of sorption sites occupied by columns of $i$ molecules for different sorbate-surface interaction strengths and different concentrations of the sorbate (see Fig. 7), for the same temperature interval $(k T=1.3$ and $k T=2)$.

In summary, multilayer adsorption simulation results are properly described by the GAB model. The description of the system with two parameters $(f$ and $c)$ allows one to interpret them in terms of fundamental magnitudes as energy and entropy of different sorbate states. Moreover, the activity coefficient of the simulated solution can also be also calculated fol- 

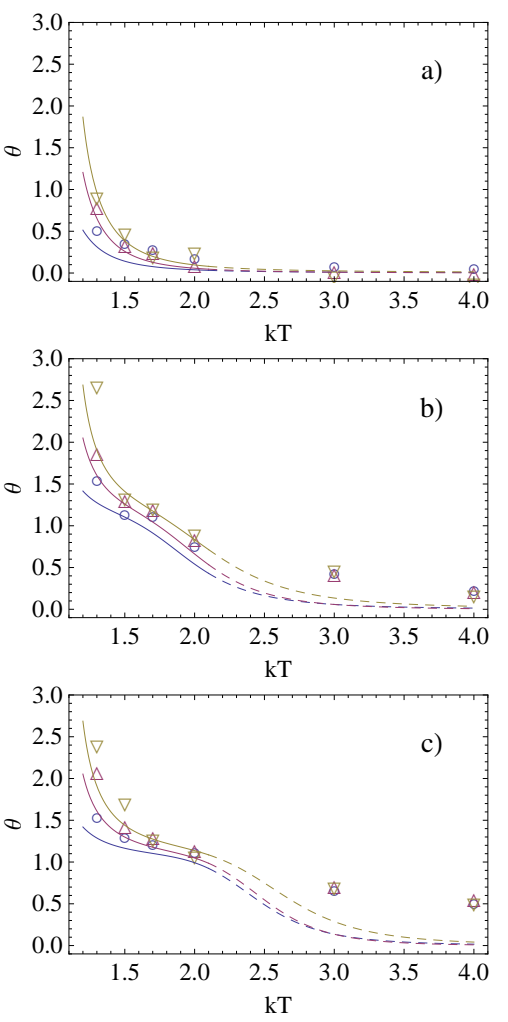

Figure 6: Adsorbed fraction $\theta$ from simulation (symbols), GAB model (lines) and extrapolation (dashed lines). Different interaction parameters $\varepsilon=1$ (a), 3 (b) and 5 (c), with different concentrations 0.1 (blue), 0.3 (purple) and 0.5 (green) are included. 

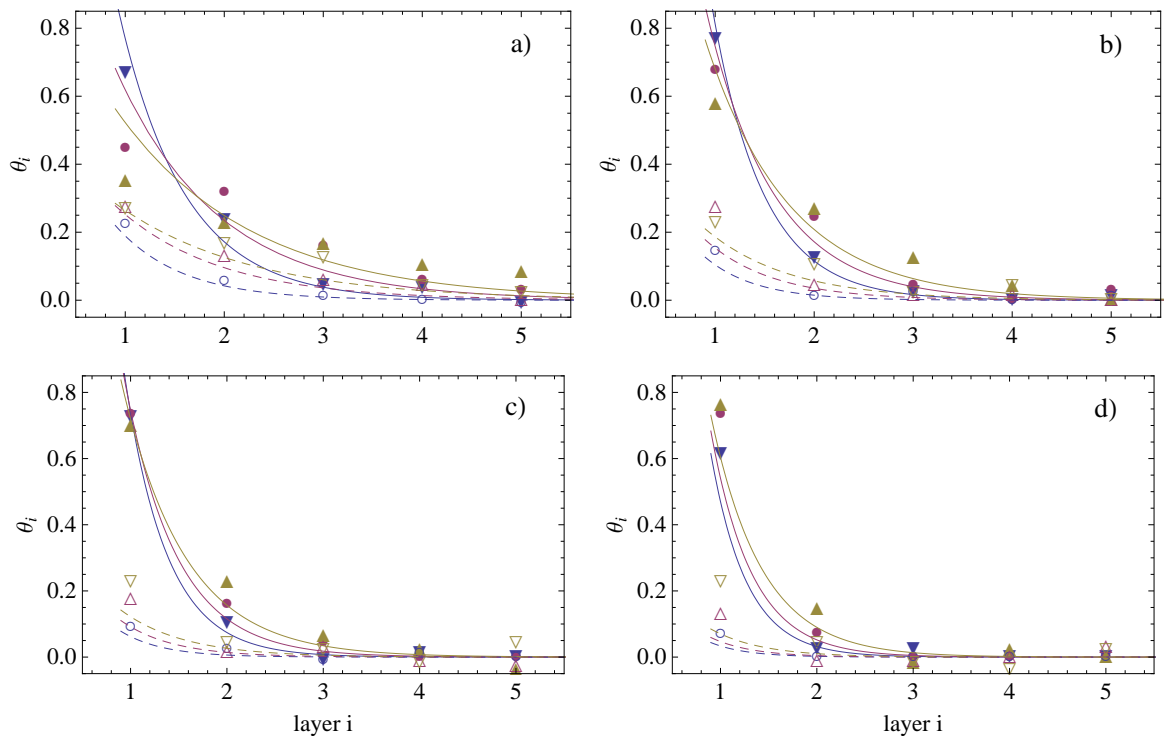

Figure 7: Fraction number of sorption sites occupied by a column of $i$ molecules from simulation (symbols) and GAB model (lines). Two interaction parameter $\varepsilon=1$ (dashed lines and open symbols) and $\varepsilon=3$ (full lines and symbols); at different temperatures $k T=1.3(\mathrm{a}), 1.5$ (b), 1.7 (c) and 2 (d); and concentrations 0.1 (blue), 0.3 (purple) and 0.5 (green) are included. 
lowing the described methodology.

\section{Conclusions}

Multilayer adsorption of simple elements on a plane surface has been simulated with the Bond Fluctuation Model, for different values of the sorbatesurface interaction, different concentrations and temperatures. At low enough temperatures $(k T \leq 1)$, adsorption occurs but the system did not reach equilibrium during the simulation period. At $k T=1$ multilayer adsorption of 8 or more layers was found, which was interpreted as a crystallization phenomenon induced by the adsorbing surface. At higher temperatures, the system reached equilibrium, which allows multilayer adsorption to be described in terms of the GAB model, for different values of the sorbate-surface interaction strength $\varepsilon$. For $\varepsilon=1$, the interaction strength is the same as the sorbate-sorbate interaction, and the first layer is not completed at equilibrium. For lower interaction strength $(\varepsilon=0.5)$ there is no adsorption, while for higher interaction $(\varepsilon=3)$ the first layer is completed.

The GAB model for multilayer adsorption reproduces the simulation results at equilibrium or moderate temperatures $(k T<3)$. The comparison between simulation data and the GAB model allows one to calculate the values of GAB parameters $c_{0}$ and $f_{0}$ as well as the activity of different solutions. The calculated parameters $c_{0}$ and $f_{0}$ were far from unity and can be used to estimate the entropy of sorbate at different states.

The interpretation of the simulation results with the model also helps to understand the meaning of the model parameters in terms of basic physical concepts and can be used to explore the limits of the model on more complex 
systems such as the adsoption of polymer chains.

\section{Acknowledgments}

The authors would like to acknowledge the support provided by the Conselleria d'Educació of the Generalitat Valenciana through the GV/2009/033 project.

\section{References}

[1] J. Pouchly, B. Benes, Z. Masa, J. Biros, Makromol. Chem. 183 (1982) 1565.

[2] E. O. Timmermann, J. Chem. Soc., Faraday Trans. 85 (1989) 1631.

[3] J. C. Galin, M. Galin, J. Polym. Sci., Part B: Polym. Phys. 30 (1992) 1113.

[4] P. P. Lewicki, Int. J. Food Sci. Technol. 32 (1997) 553.

[5] G. Velázquez, A. Herrera-Gómez, M. O. Martín-Polo, J. Food. Eng. 59 (2003) 45 .

[6] S. Brunauer, P. H. Emmett, E. Teller, J. Am. Chem. Soc. 60 (1938) 309.

[7] R. B. Anderson, J. Am. Chem. Soc. 68 (1946) 686.

[8] E. A. Guggenheim, Applications of Statistical Mechanics, Clarendon, Oxford, 1966. 
[9] A. W. Adamson, Physical Chemistry of Surfaces, Wiley, New York, 1990.

[10] S. R. Fowler, E. A. Guggenheim, Statistical Thermodynamics, Cambridge University Press, Cambridge, 1949.

[11] C. Kemball, G. D. L. Schreiner, J. Am. Chem. Soc. 72 (1950) 5605.

[12] H. Naono, M. Hakuman, J. Colloid Interface Sci. 145 (1991) 405.

[13] A. Tsiapouris, L. Linke, Starch 2 (2000) 53.

[14] D. Zeng, W. Voight, Calphad 27 (2003) 243.

[15] M. Monleón Pradas, M. Salmerón Sánchez, G. Gallego Ferrer, J. L. Gómez Ribelles, J. Chem. Phys. 121 (2004) 8524.

[16] I. Carmesin, K. Kremer, Macromolecules 21 (1988) 2819.

[17] K. Binder, Monte Carlo and Molecular Dynamics Simulations in Polymer Science, Oxford University Press, New York, Oxford, 1995.

[18] A. Koutsioubas, N. Spiliopoulos, D. Anastassopoulos, A. Vradis, C. Toprakcioglu, J. Chem. Phys. 131 (2009) 44901.

[19] P. Lai, Phys. Rev. E 49 (1994) 5420.

[20] J. Semler, J. Genzera, J. Chem. Phys. 119 (2003) 5274.

[21] H. Deutsch, K. Binder, J. Chem. Phys. 94 (1990) 2294. 